\title{
Erratum to: Pruned Bi-directed K-nearest Neighbor Graph for Proximity Search
}

\author{
Masajiro Iwasaki ${ }^{(凶)}$ \\ Yahoo Japan Corporation, Tokyo, Japan \\ miwasaki@yahoo-corp.jp
}

Erratum to:

Chapter 2 in: L. Amsaleg et al. (Eds.)

Similarity Search and Applications

DOI: 10.1007/978-3-319-46759-7_2

In an older version of the paper starting on p. 20 of the SISAP proceedings (LNCS 9939), Fig. 5(b) was represented incorrectly. This has been corrected.

The updated original online version for this Chapter can be found at DOI: 10.1007/978-3-319-46759-7_2 